\title{
Quiste dermoide con defecto óseo en apófisis cigomática del frontal. Reporte de un caso
}

\section{Dermoid cyst with bone defect in the frontal zygomatic process. A case report}

\author{
Juliana M. Morales-Avalos ${ }^{1 *}$, Adolfo J. Torres-Moreno ${ }^{2}$, Jesus A. Cortez-Hernandez ${ }^{3}$ y \\ Hiram J. Arce-Sanchez ${ }^{4}$ \\ ${ }^{1}$ Médico Oftalmólogo Oculoplástico, Servicio de Oftalmología, Hospital General de Culiacán «Dr. Bernardo J. Gastelum», Culiacán Rosales; ${ }^{2}$ Médico \\ Oftalmólogo del Segmento Anterior, Servicio de Oftalmología, Hospital General de Culiacán «Dr. Bernardo J. Gastelum», Culiacán Rosales; ${ }^{3}$ Médico \\ Patólogo, Jefe del Departamento de Patología, Hospital de la Mujer SSA, Culiacán Rosales; ${ }^{4}$ Médico Radiólogo, Jefe del Departamento de \\ Radiología, Hospital General de Culiacán «Dr. Bernardo J. Gastelum», Culiacán Rosales. Sinaloa, México
}

\section{Resumen}

Paciente mujer de 23 años, que presenta aumento de volumen en la región frontocigomática derecha, detectada desde la infancia, se realizan estudios de tomografía axial computarizada y topografía helicoidal multiforme, identificando una lesión quística sobre la apófisis cigomática del frontal, con un orificio de espesor completo del cual emergía la lesión. Se sometió a resección quirúrgica, confirmando por patología el diagnóstico de quiste dermoide; la evolución ha sido satisfactoria. Se presenta el presente caso debido a la rareza del defecto óseo acompañante, dado que generalmente se asocia a una localización sobre la sutura frontocigomática.

Palabras clave: Quiste dermoide orbitario. Abordaje en pliegue palpebral. Localización inusual. Defecto óseo. Caso clínico.

\section{Abstract}

A 23 old female, with a mass in the right zigomaticofrontal region beginning from the childhood. Imaging tests show a cyst on the frontal zigomatic process with a total thickness bone defect under the cyst. Surgical resection was performed. The histopathologic examination resulted in dermoid cyst. The monitoring has been satisfactory. We present this case due to the unusual bone defect because usually the orbital dermoid cyst is located on the frontozygomatic suture.

Key words: Orbital dermoid cyst. Eyelid crease approach. Unusual location. Bone defect. Clinical case.

\section{Correspondencia:}

*Juliana M. Morales-Avalos

E-mail: marzzela@ hotmail.com
Disponible en internet: 03-08-2016

Rev Mex Oftalmol. 2018;xx(x):1-4 www.rmo.com.mx

(87-4519/C 2016 Sociedad Mexicana de Oftalmología. Publicado por Permanyer México SA de CV. Este es un artículo Open Access bajo la licencia CC BY-NC-ND (http://creativecommons.org/licenses/by-nc-nd/4.0/) 


\section{Introducción}

Los dermoides son catalogados dentro del grupo de coristomas, los cuales son lesiones congénitas originadas de tejido ectodérmico aberrante ${ }^{1,2}$. Se presentan generalmente al momento de cierre embrionario entre 2 líneas de suturas craneales, atrapando tejido dérmico y subdérmico, formando un quiste ${ }^{3}$. El $50 \%$ de los dermoides en cráneo se encuentran en la órbita ${ }^{4,5}$. No tiene predilección por raza o género ${ }^{6}$. Su presentación clínica es la presencia de una masa indolora orbitaria de crecimiento lento, las localizaciones orbitarias profundas pueden presentar diplopía y proptosis ${ }^{7}$. Las localizaciones más comunes en orden de frecuencia son la temporal superior y la nasal superior ${ }^{8-10}$. Puede presentarse rotura del quiste espontánea o traumática con una reacción inflamatoria intensa que puede imitar una celulitis orbitaria ${ }^{11-13}$.

\section{Presentación del caso}

Mujer de 23 años, que acude al servicio de oftalmología con presencia de aumento de volumen en la región frontocigomática derecha, identificada desde la infancia, que presentó crecimiento progresivo, indolora, que no condicionaba otra sintomatología (figs. 1A y B). Se realizó la biomicroscopia sin hallazgos patológicos, identificamos una masa en área de la pared lateral de la órbita derecha, de bordes definidos, indolora a la palpación, fija a planos profundos, de consistencia firme de $2 \square 2 \mathrm{~cm}$. Realizamos tomografía axial computarizada (TAC) de orbitas y topografía helicoidal multiforme, valorando una masa hipodensa con una zona central hiperdensa en región de pared lateral de la órbita derecha, en contacto con el músculo temporal hacia su margen posterior de $23 \mathrm{~mm}$ de longitud vertical, $17 \mathrm{~mm}$ de longitud vertical y $10 \mathrm{~mm}$ de espesor, que no reforzó con la administración de medio de contraste. Observando también el defecto óseo en el espesor de la apófisis cigomática del frontal derecho al cual estaba adherida la lesión quística (figs. $2 \mathrm{~A}, \mathrm{~B}, \mathrm{C}$ y D).

Se realizó cirugía de exéresis de lesión, mediante una incisión de blefaroplastia superolateral, disecando e identificando la lesión, logrando resecar en su totalidad, el defecto óseo de $3 \mathrm{~mm}$ de diámetro se identificó cubierto por periostio en su sector posterior y se realizó cierre de herida por planos (figs. $3 \mathrm{~A}$ y B). En el estudio histopatológico se confirmó el diagnóstico de quiste dermoide orbitario (fig. 4). Actualmente la paciente cursa con adecuada evolución a 8 meses de la cirugía.

\section{Discusión}

La mayoría de los casos de dermoides de pared lateral orbitaria se asocian a la sutura frontocigomática ${ }^{1}$, sin embargo este caso se asoció a una ubicación más superior en la apófisis cigomática del hueso frontal, condicionando la presencia de un defecto óseo subyacente de espesor completo.

\section{Conclusión}

Es importante identificar las lesiones orbitarias, realizar una adecuada anamnesis del padecimiento y utilizar los recursos paraclínicos que tengamos disponibles para identificar la naturaleza de las lesiones, y ofrecer el tratamiento adecuado. Este tipo de

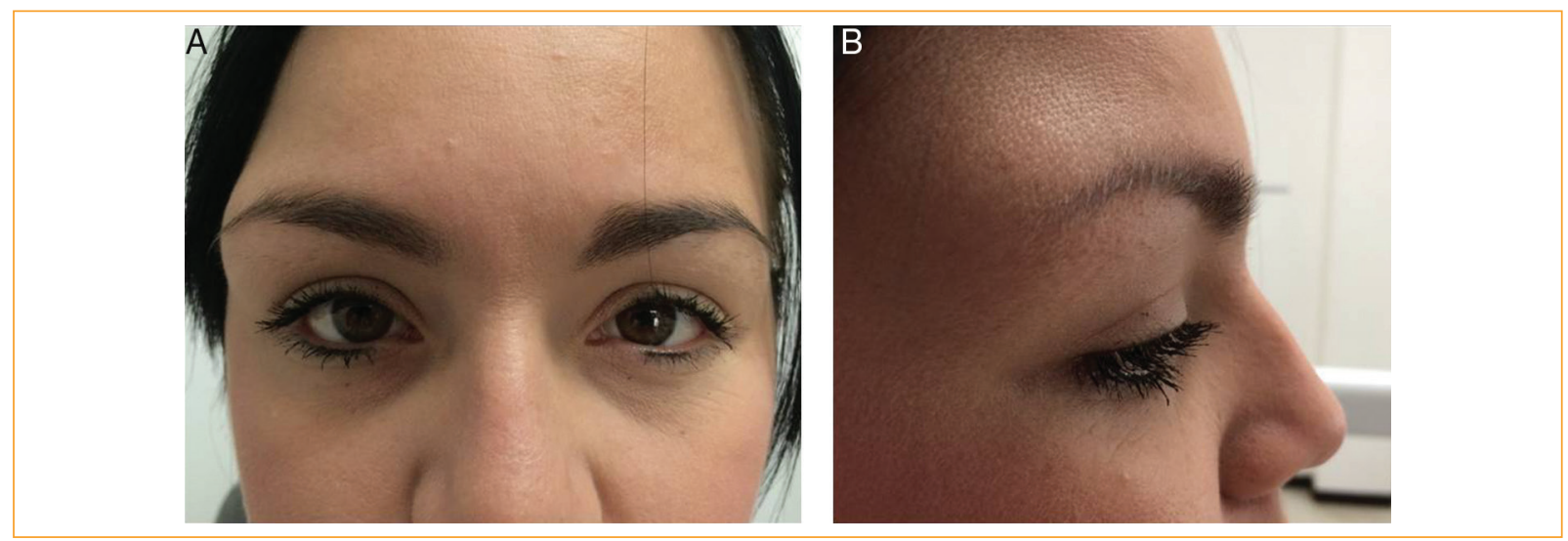

Figura 1. A: Vista frontal. B: Lateral derecha. 

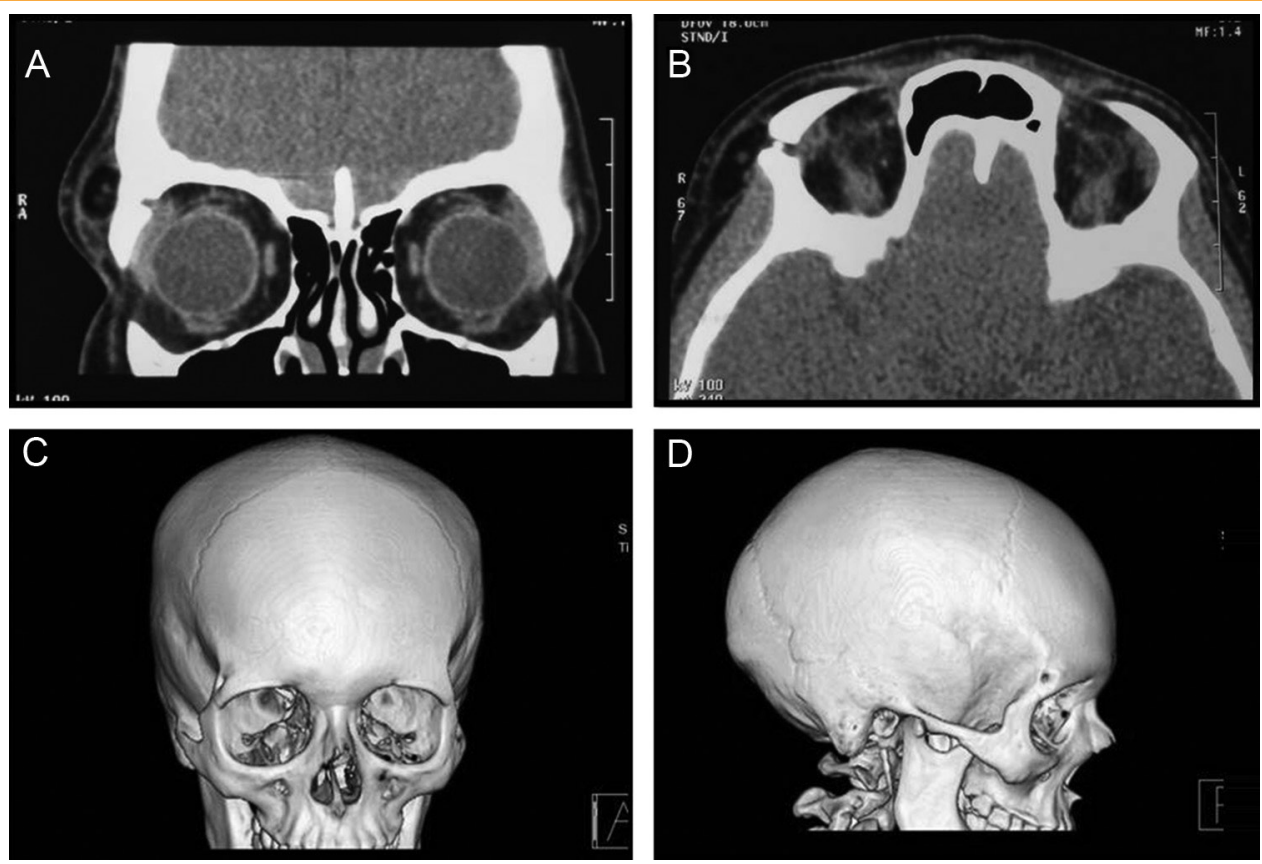

Figura 2. A-D: Tomografía axial corte coronal, tomografía axial corte axial de orbitas. Topografía helicoidal vista frontal y topografía helicoidal vista lateral derecha.

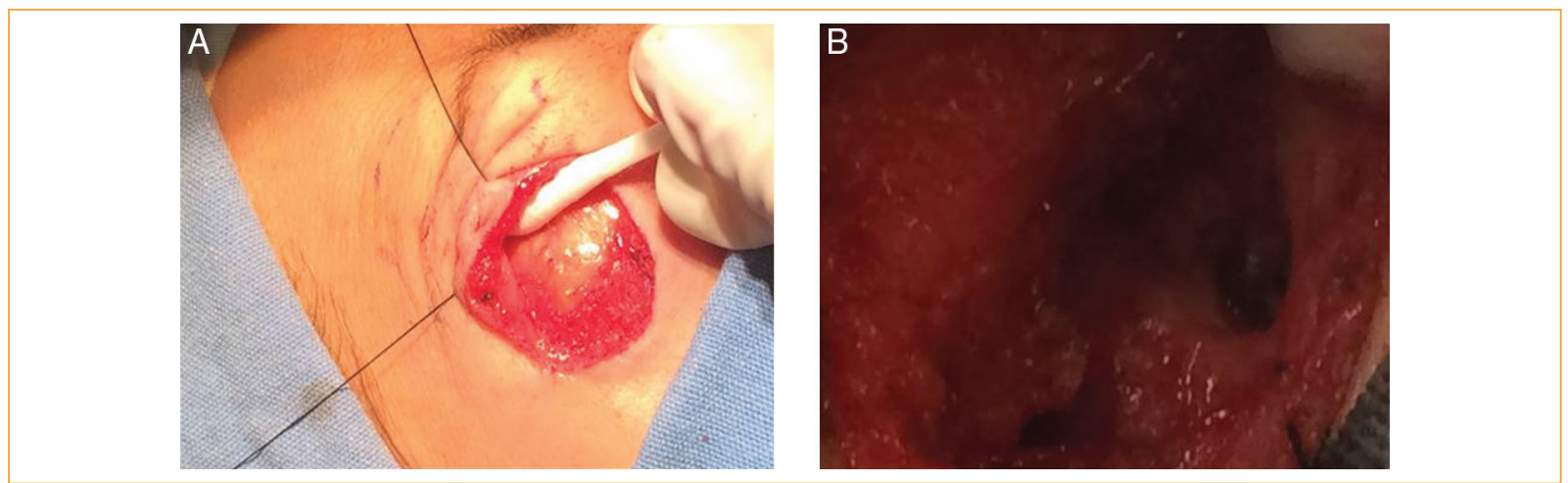

Figura 3. A, B: Disección de quiste dermoide vía blefaroplastia superior, defecto óseo frontal.

coristomas es común en nuestro centro de trabajo, recibimos a este caso con un trayecto óseo inusual que generó controversia en el aspecto de que el defecto óseo fuera causado por un proceso de erosión, sin embargo, comprende el espesor completo del hueso frontal, por lo que sugiere ser el trayecto de atrapamiento de tejidos ectodérmicos durante la formación embrionaria.

\section{Responsabilidades éticas}

Protección de personas y animales. Los autores declaran que para esta investigación no se han realizado experimentos en seres humanos ni en animales.

Confidencialidad de los datos. Los autores declaran que han seguido los protocolos de su centro de trabajo sobre la publicación de datos de pacientes.

Derecho a la privacidad y consentimiento informado. Los autores declaran que en este artículo no aparecen datos de pacientes.

\section{Financiamiento}

Los autores no recibieron patrocinio para llevar a cabo este artículo. 


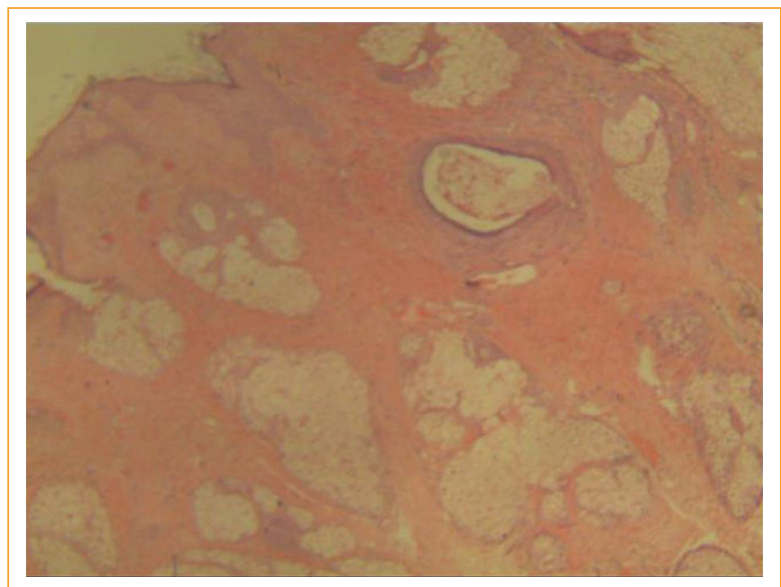

Figura 4. Panorámica de lesión quística con revestimiento por epitelio escamoso con queratinización. Tinción hematoxilina eosina.

\section{Conflicto de intereses}

Los autores declaran no tener ningún conflicto de intereses.

\section{Bibliografía}

1. Perry JD, Tuthill R. Simultaneous ipsilateral temporal fossa andorbital dermoid cysts. Am J Ophthalmol. 2003;135:413-5.

2. Vega RA, Hidlay DT, Tye GW, Fuller CE, Rhodes JL. Intradiploicdermoid cyst of the lateral frontotemporal skull: case reportand review of the literature. Pediatr Neurosurg. 2013;49:232-5.

3. Sherman RP, Rootman J, Lapointe JS. Orbital dermoids: Clinical presentation and management. Br J Ophthalmol. 1984;68:642-52.

4. Golden BA, Jaskolka MS, Ruiz RL. Craniofacial and orbitaldermoids in children. Oral Maxillofac Surg Clin North Am.2012;24:417-25.

5. Ruszkowski A, Caouette-Laberge L, Bortoluzzi P, Egerszegi EP. Superior eyelid incision: An alternative approach for frontozy-gomatic dermoid cyst excision. Ann Plast Surg. 2000;44:591-4.

6. Kersten RC. The eyelid crease approach to superficiallateral dermoid cysts. J Pediatr Ophthalmol Strabismus.1988;25:48-51.

7. Nelson KE, Mishra A, Duncan C. Upper blepharoplastyapproach to frontozygomatic dermoid cysts. J Craniofac Surg. 2011;22:e41-4.

8. Ortube MC, Dipple K, Setoguchi Y, Kawamoto HK Jr, Demer JL. Ocular manifestations of oblique facial clefts. J Craniofac Surg. 2010;21:1630-1.

9. Nevrekar D, Abdu E, Selden NR. Craniectomy for a bilobed der-moid cyst in the temporal fossa and greater wing of the sphenoidbone. Pediatr Neurosurg. 2009;45:46-8.

10. Meyer DR, Lessner AM, Yeatts RP, Linberg JV. Primary temporalfossa dermoid cysts. Characterization and surgical manage-ment. Ophthalmology. 1999;106:342-9.

11. Scolozzi P, Lombardi T, Jaques B. Congenital intracranial fronto-temporal dermoid cyst presenting as a cutaneous fistula. Head Neck. 2005;27:429-32.

12. Parag P, Prakash PJ, Zachariah N. Temporal dermoid-an unusualpresentation. Pediatr Surg Int. 2001;17:77-9.

13. Sathananthan N, Moseley IF, Rose GE, Wright JE. The frequencyand clinical significance of bone involvement in outer canthusdermoid cysts. Br J Ophthalmol. 1993;77:789-94. 